\title{
$\beta$-Adrenergic receptor gene polymorphism is a genetic risk factor for cardiovascular disease: a cohort study with hypertensive patients
}

\author{
Yoshihiro Iwamoto ${ }^{1,3}$, Mitsuru Ohishi ${ }^{1}$, Ming Yuan ${ }^{1,2,3}$, Yuji Tatara ${ }^{1}$, Nozomi Kato ${ }^{1}$, Yasushi Takeya ${ }^{1}$, \\ Miyuki Onishi ${ }^{1}$, Yoshihiro Maekawa ${ }^{1}$, Kei Kamide ${ }^{1}$ and Hiromi Rakugi ${ }^{1}$
}

Single-nucleotide polymorphisms (SNPs) of the $\beta$-adrenergic receptor ( $\beta A D R)$ subtypes are related to hypertension and obesity. This hospital-based cohort study with hypertensive patients evaluated five $\beta A D R$ SNPs in association with cardiovascular events. The cohort included 357 hypertensive patients (male $=181$; mean age $=61.5 \pm 11.8$ years) seen between January 1998 and June 2004. The SNPs (Ser49Gly and Arg389Gly for $\beta_{1} A D R$; Gly16Arg and Glu27GIn for $\beta_{2} A D R$; Trp64Arg for $\beta_{3} A D R$ ) were identified by PCR. We used Kaplan-Meier curves to assess the prognostic effect of these SNPs on cardiovascular disease (CVD). The SNP frequencies were Ser/Ser:Ser/Gly:Gly/Gly=243:104:10; Arg/Arg:Arg/Gly:Gly/Gly=256:95:6; Gly/Gly:Gly/Arg:Arg/ Arg=71:201:85; Gln/Gln:Glu/Gln=308:49; and Trp/Trp:Trp/Arg:Arg/Arg=265:89:3. A total of 17 stroke and 15 coronary artery disease cases were recorded. By Kaplan-Meier analysis, the Ser/Ser SNP in Ser49Gly $(P=0.0398)$, the Glu/Gln SNP in Glu27GIn $(P=0.0390)$ and the Trp/Trp SNP in Trp64Arg $(P=0.0132)$ were associated with lower event-free CVD survival (log-rank, Mantel-Cox model). A Cox proportional hazards model revealed that only the $\operatorname{Trp} / \operatorname{Trp}$ SNP $(\boldsymbol{P}=\mathbf{0 . 0 3 2 1 )}$ and age $(P=0.0186)$ were independently related to lower event-free survival for CVD, adjusted for gender, diabetes, dyslipidemia, blood pressure, body mass index, medication and hypertensive complications. Combination Kaplan-Meier analysis of these three positive SNPs indicated a higher frequency of CVD among patients with the combination of Ser/Ser in Ser49Gly of $\beta_{1}$, Glu/Gln in Glu27GIn of $\beta_{2}$ and Trp/Trp in Trp64Arg of $\beta_{3}(P=0.0209)$. These three SNPs, especially the Trp64Arg SNP of $\beta_{3} A D R$, might be risk factors for CVD in hypertensive patients.

Hypertension Research (2011) 34, 573-577; doi:10.1038/hr.2010.281; published online 3 February 2011

Keywords: $\beta$-adrenoreceptor; cohort study; hypertensive complication; polymorphism

\section{INTRODUCTION}

The human $\beta$-adrenergic receptor $(\beta A D R)$ is a member of the family of seven-transmembrane G-protein-coupled receptors and is encoded by a gene on chromosome $5 .^{1}$ Previous studies have shown that sympathetic nervous system activity by $\beta$ ADRs has an important role in the development of hypertension and its complications. ${ }^{2}$ Three isotypes of human $\beta A D R, \beta_{1}, \beta_{2}$ and $\beta_{3}$, are involved in this system. The classical subdivision of $\beta A D R$ s defines $\beta_{1}$ as the subtype that stimulates cardiac muscle ${ }^{3}$ and $\beta_{2}$ as the subtype that relaxes smooth muscle. ${ }^{4}$ The expression of the $\beta_{3}$ subtype is essentially limited to adipose tissue. ${ }^{5}$ A number of single-nucleotide polymorphisms (SNPs) of the $\beta A D R$ subtypes have recently been reported to be positional candidate genes for cardiovascular diseases (CVDs). ${ }^{6}$ For instance, the genotypes of the Ser49Gly and Arg389Gly polymorphisms in the human $\beta_{1} A D R$ gene have been reported to be associated with arterial stiffness ${ }^{7}$ and acute myocardial infarction, ${ }^{8}$ respectively. An association study ${ }^{9}$ has suggested that the gene encoding $\beta_{2} A D R$ is associated with essential hypertension, and the Arg64 allele of the $\beta_{3} A D R$ gene is associated with obesity-related phenotypes, insulin resistance, hypertension, coronary artery disease and earlier age of onset of diabetes. ${ }^{10}$ As noted, many studies have reported cross-sectional effects of these SNPs, and several cohort studies ${ }^{11}$ have focused on one of these SNPs, but no cohort study on SNPs of $\beta A D R$ subtypes has focused on evaluating cardiovascular morbidity.

The aim of the present hospital-based cohort study, therefore, was to investigate the potential prognostic impact of the $\beta A D R$ SNPs that result in amino acid substitutions on the occurrence of CVDs and stroke in patients with essential hypertension.

\section{METHODS}

Study population and study design

This hospital-based cohort study was designed as a part of the NOn-invasive Atherosclerotic evaluation in Hypertension study. ${ }^{12}$ In the NOn-invasive Atherosclerotic evaluation in Hypertension study, 813 serial outpatients who had been diagnosed with essential hypertension were sequentially recruited between January 1998 and June 2004 at the Osaka University Hospital. The

\footnotetext{
${ }^{1}$ Department of Geriatric Medicine, Osaka University Graduate School of Medicine, Osaka, Japan and 2Department of Cardiology, Xijing Hospital, Xi'an Shaanxi, China 3These authors contributed equally to this work.

Correspondence: Dr M Ohishi, Department of Geriatric Medicine, Osaka University Graduate School of Medicine, 2-2 Yamadaoka, Suita, Osaka 565-0871, Japan. E-mail: ohishi@geriat.med.osaka-u.ac.jp

Received 9 September 2010; revised 4 November 2010; accepted 18 November 2010; published online 3 February 2011
} 
study protocol was approved by the hospital ethics committee, written informed consent was obtained from all participants, and additional written informed consent was obtained from 384 participants for the analysis of genotypes; seven patients opted not to participate in this study. A clinical survey was conducted for every patient, and 14 patients were excluded because of insufficient information about cardiovascular events and/or mortality. We also evaluated five representative $\beta A D R$ SNPs and could not detect these SNPs in 13 patients. Ultimately, our analysis included 357 hypertensive patients, as shown in Table 2. A total of 120 patients were not treated with any anti-hypertensive drugs, and 237 patients were treated with one or more anti-hypertensive drugs: 137 patients with a calcium antagonist, 90 patients with an angiotensin II receptor blocker, 69 patients with an angiotensinconverting enzyme inhibitor, 45 patients with a $\beta$-blocker, 23 patients with a diuretic and 20 patients with an $\alpha$-blocker. In addition, 110 patients were also treated with a statin.

\section{Follow-up evaluation}

Clinical follow-up was conducted by clinical visits, mailed questionnaires and telephone contact every September from 2003. The questionnaire included events related to hypertensive complications (described below) and cause of death. We also confirmed responses in detail by comparing them against patient medical records. The primary endpoint of this study was stroke (paralysis and diagnosis with computed tomography and/or magnetic resource imaging); the onset of CVD, including angina pectoris (typical chest pain with ST segment changes of electrocardiogram), myocardial infarction (St segmental elevation with more than twofold elevation of creatinine kinase), or heart failure (diagnosed by American Heart Association criteria); or the rupture of an aortic aneurysm (diagnosed by ultrasound echography or computed tomography). The follow-up duration was considered to encompass the interval from the initial evaluation to the time of event onset or September 2006. The average follow-up period was $57.0 \pm 23.7$ months.

Table 1 Patient characteristics and $\beta$-adrenoceptor polymorphisms at baseline

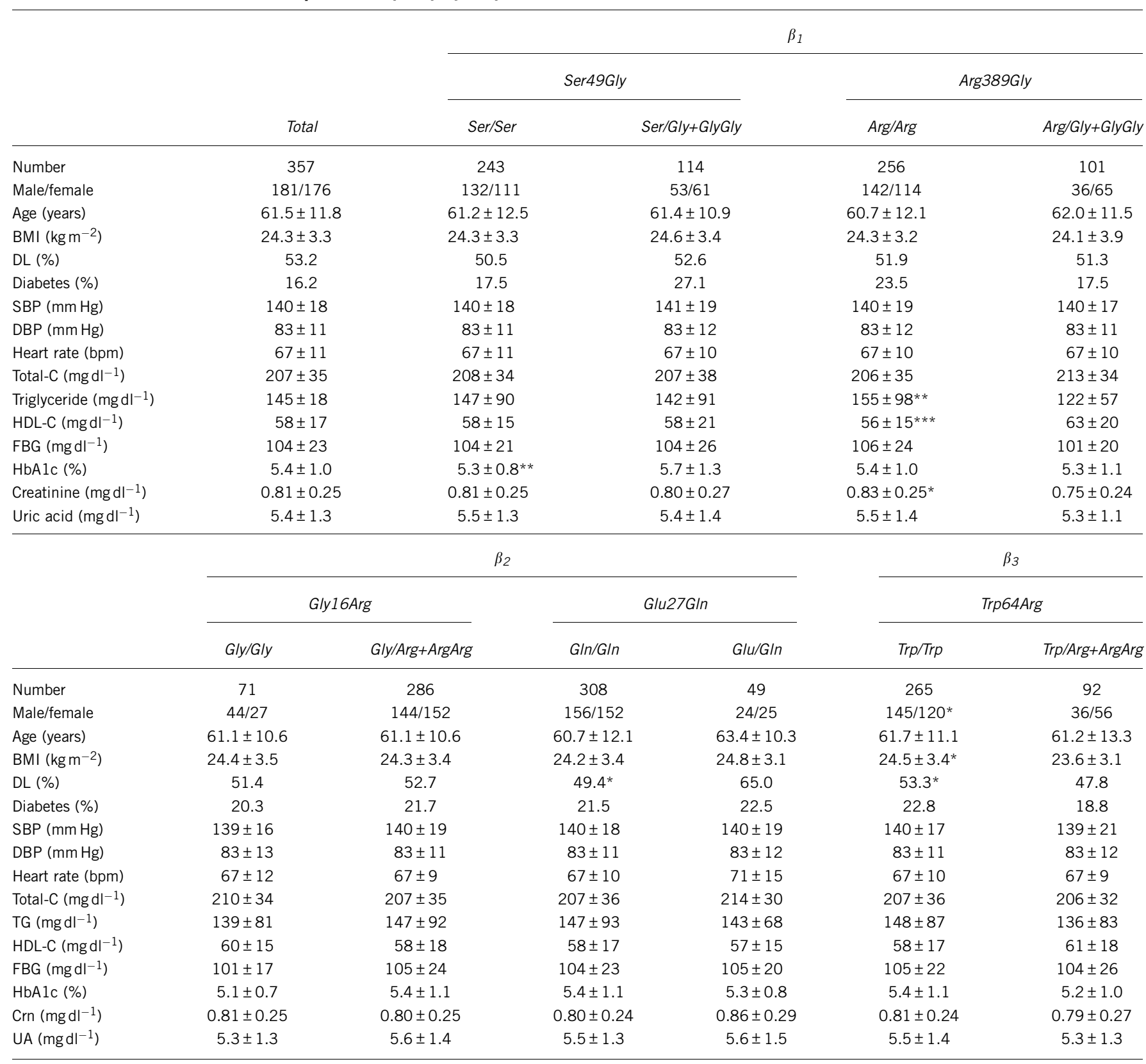

Abbreviations: BMI, body mass index; Crn, creatinine; DBP, diastolic blood pressure; DL, dyslipidemia; FBG, fasting blood glucose; HDL-C, high-density lipoprotein cholesterol; SBP, systolic blood pressure; Total-C, total cholesterol; TG, triglyceride; UA, uric acid. ${ }^{*} P<0.05,{ }^{* *} P<0.01,{ }^{* * *} P<0.001$ vs. other genotype. 


\section{Genotyping}

Total genomic DNA was extracted from leukocytes obtained from samples of whole blood, following standard techniques. In this study, the TaqMan PCR assay as described previously was used to perform polymorphism analysis of the three $\beta A D R s, \beta_{1}, \beta_{2}$ and $\beta_{3}{ }^{7}$. We detected two polymorphisms of $\beta_{1} A D R$ that result in serine/glycine (Ser49Gly) and arginine/glycine (Arg389Gly) amino acid substitutions at residues 49 and 389, respectively. We also detected two polymorphisms of $\beta_{2} A D R$ that result in glycine/arginine (Gly16Arg) and glutamate/glutamine (Glu27Gln) amino acid substitutions at residues 16 and 27, respectively. Finally, we identified a $\beta_{3} A D R$ polymorphism that results in a tryptophan/arginine (Trp64Arg) amino acid substitution at residue 64 .

\section{Statistical analysis}

Data were analyzed using JMP ver. 4 (SAS, Cary, NC, USA) and are presented as the mean \pm s.d. in Table 1 . An event-free curve was estimated by the KaplanMeier method. Patients were stratified according to SNPs for the analysis of event-free rates. The log-rank test was used to compare the differences in event-free rates among these patient groups. Baseline clinical variables for these patients were analyzed with the Cox proportional hazards model, and the hazard ratio with a $95 \%$ confidence interval for each factor is given. Backward elimination techniques were used to identify variables independently associated with event onset. Analysis of variance and the Student's $t$-test were used to test for significant differences among the SNPs. A value of $P<0.05$ was regarded as statistically significant.

\section{RESULTS}

Table 1 presents the genotype frequencies and participant characteristics stratified by genotypes. With respect to the Gly16Arg $\beta_{2} A D R$ polymorphism, systolic blood pressure values in patients with the Gly16Gly or Gly16Arg polymorphism were significantly lower than those of patients with the Arg16Arg polymorphism. With respect to the Trp64Arg $\beta_{3} A D R$ polymorphism, the body mass index values of patients with the Arg64Arg or Trp64Arg polymorphism were significantly higher than those of patients carrying other genotypes.

We classified the SNPs into pairs: SerGly+GlyGly and SerSer for Ser49Gly $\beta_{1} A D R$; ArgGly+GlyGly and ArgArg for Arg389Gly $\beta_{1} A D R$; ArgArg+ArgGly and GlyGly for Gly16Arg $\beta_{2} A D R$; GlnGln and GluGln for Glu27Gln $\beta_{2} A D R$; and ArgArg+TrpArg and TrpTrp for Trp64Arg $\beta_{3} A D R$. Figure 1 shows the results of the Kaplan-Meier analysis for event-free survival with respect to CVD and stroke classified by SNP group. Patients with SerSer in the Ser49Gly $\beta_{1} A D R$ polymorphism showed lower event-free survival compared with those with SerGly and GlyGly polymorphisms ( $P=0.0398$ ) (Figures $1 \mathrm{a}$ and $1 \mathrm{~b}$ ); however, there was no statistical significance for the Arg389Gly $\beta_{1} A D R$ polymorphism. Analysis of $\beta_{2} A D R$ polymorphisms showed that patients with the GluGln polymorphism in Glu27Gln $\beta_{2} A D R$ had lower event-free survival compared with patients with GlnGln polymorphisms $(P=0.0390)$; however, there was no statistical significance for the Gly16Arg $\beta_{2} A D R$ polymorphism. Finally, patients with the
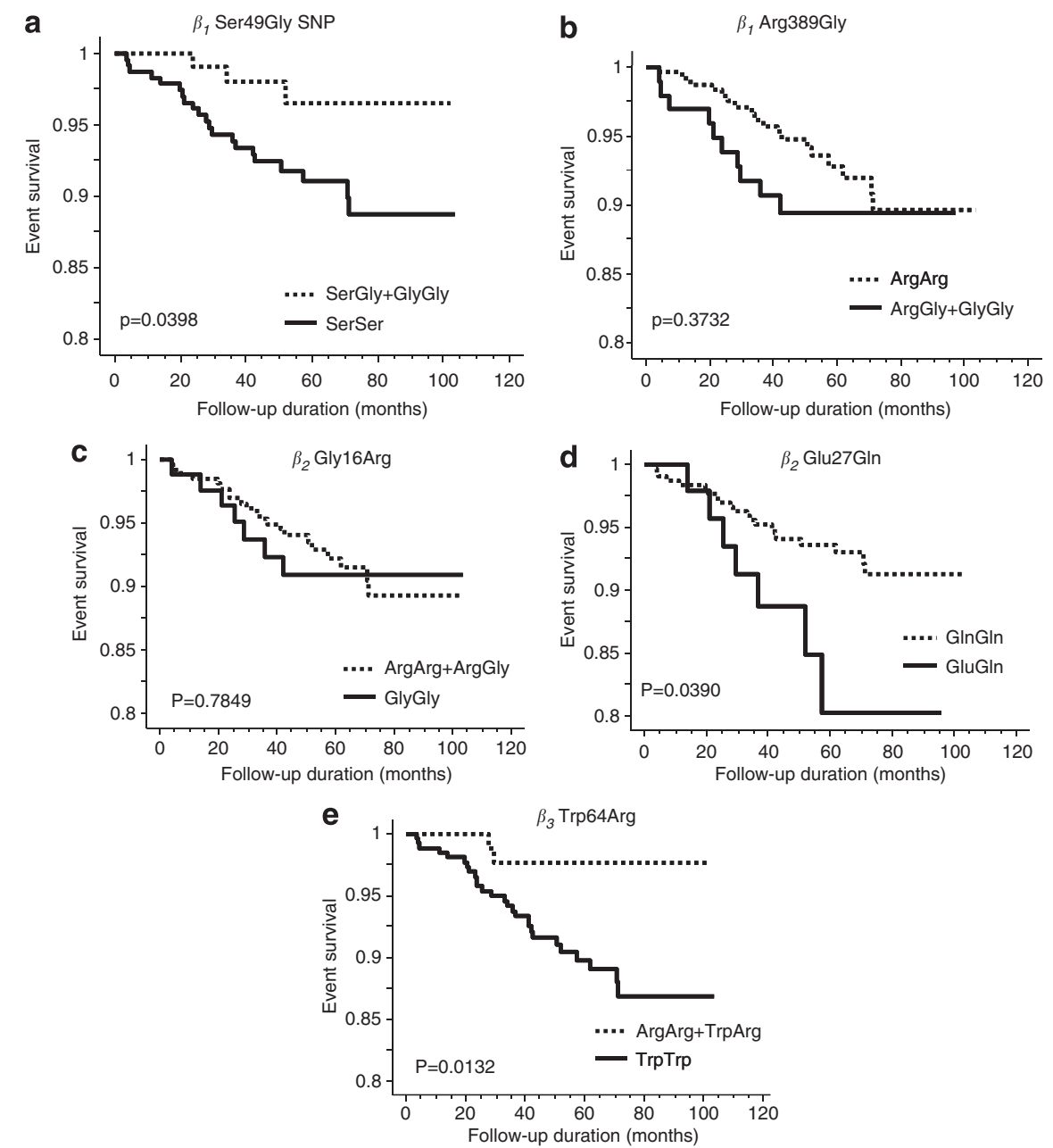

Figure 1 Kaplan-Meier analysis for stroke and CVD in each SNP group. (a) $\beta_{1}$ Ser49Gly SNP; (b) $\beta_{1}$ Arg389Gly; (c) $\beta_{2}$ Gly16Arg; (d) $\beta_{2}$ Glu27Gln; and (e) $\beta_{3}$ Trp64Arg. 
Table 2 Cox's proportional hazard model for CVD+stroke

\begin{tabular}{lccc}
\hline & Exp & $95 \%$ Cl & P-value \\
\hline Model 1 & & & \\
Gender (female) & 1.185 & $0.455-3.087$ & 0.7287 \\
Age (/years) & 1.080 & $1.023-1.141$ & 0.0052 \\
Diabetes (-) & 2.710 & $0.336-21.86$ & 0.3492 \\
Dyslipidemia (-) & 0.896 & $0.342-2.348$ & 0.8226 \\
Medication (-) & 1.112 & $0.414-2.984$ & 0.8336 \\
Systolic blood pressure & 1.007 & $0.974-1.042$ & 0.6801 \\
Diastolic blood pressure & 1.036 & $0.973-1.102$ & 0.2665 \\
Pulse rate & 0.941 & $0.508-1.032$ & 0.9059 \\
$\beta_{1}$ Ser49Gly (CC; SerSer) & 2.017 & $0.554-7.344$ & 0.2871 \\
$\beta_{2}$ Glu27GIn (CG; GInGlu) & 2.358 & $0.812-6.847$ & 0.1147 \\
$\beta_{3}$ Trp64Arg (TT; TrpTrp) & 9.067 & $1.141-72.07$ & 0.0371 \\
& & & \\
Model 2 & & & \\
Gender (female) & 1.443 & $0.429-4.855$ & 0.5532 \\
Age (/years) & 1.045 & $0.984-1.110$ & 0.1521 \\
Dyslipidemia & 1.436 & $0.430-4.794$ & 0.5562 \\
BMI & 0.925 & $0.766-1.117$ & 0.4194 \\
HbA1c & 1.790 & $1.050-3.050$ & 0.0323 \\
$\beta_{1}$ Ser49Gly (CC; SerSer) & 10.67 & $1.059-107.9$ & 0.0446 \\
$\beta_{2}$ Glu27GIn (CG; GInGlu) & 5.272 & $1.499-18.55$ & 0.0096 \\
$\beta_{3}$ Trp64Arg (TT; TrpTrp) & 4.480 & $0.512-39.22$ & 0.1755 \\
\hline A & & &
\end{tabular}

Abbreviations: $\mathrm{BMI}$, body mass index; $\mathrm{Cl}$, confidence interval; CVD, cardiovascular disease.

TrpTrp polymorphism in Trp64Arg $\beta_{3} A D R$ had a lower event-free survival compared with those with the ArgArg and ArgTrp polymorphisms $(P=0.0132)$.

To clarify the influences of other risk factors on event-free survival with respect to stroke and CVD, we used the Cox's proportional hazards model (Table 2). In model 1, which incorporated common risk factors as confounding factors, TrpTrp in Trp64Arg $\beta_{3} A D R$ SNP emerged as an independent risk factor along with age. In model 2, we analyzed several confounding factors that showed statistical positivity $(P<0.05)$ in the analysis of patient characteristics and a $\beta A D R$ SNP at baseline. In this model, the SerSer polymorphism in Ser49Gly $\beta_{1} A D R$ and the GluGln polymorphism in Glu27Gln $\beta_{2} A D R$ emerged as independent risk factors in addition to age and HbAlc values.

We also used a combined Kaplan-Meier analysis incorporating the Ser49Gly $\beta_{1} A D R$, Glu27Gln $\beta_{2} A D R$ and Trp64Arg $\beta_{3} A D R$ SNPs (Figure 2). The shape of the Kaplan-Meier curve for each $\beta A D R$ SNP indicated statistical significance $(P=0.0209)$. In patients with the SerSer polymorphism in the Ser49Gly $\beta_{1} A D R$, the GluGln polymorphism in the Glu27Gln $\beta_{2} A D R$ and the TrpTrp polymorphism in the Trp64Arg $\beta_{3} A D R$, the event-free survival ratio was the lowest relative to the other combined SNPs.

\section{DISCUSSION}

In this hospital-based study, the frequencies of five SNPs of three $\beta A D R$ s in a patient population were determined, and the frequencies of each SNP were similar to those identified in previous reports. ${ }^{13-15}$ Several functional analyses have addressed these SNPs; for example, participants with the Gly allele in the Ser49Gly polymorphism of $\beta_{1} A D R$ have been reported to have a lower heart rate. ${ }^{16}$ In vitro studies of isoproterenol stimulation showed that Arg389 in $\beta_{1} A D R$ produces higher levels of adenylyl cyclase activity, resulting in enhanced cardiac sensitivity to catecholamines. ${ }^{17}$ The Gly16 and Glu27 polymorphisms in $\beta_{2} A D R$ have been associated with sympathetic overactivity, as

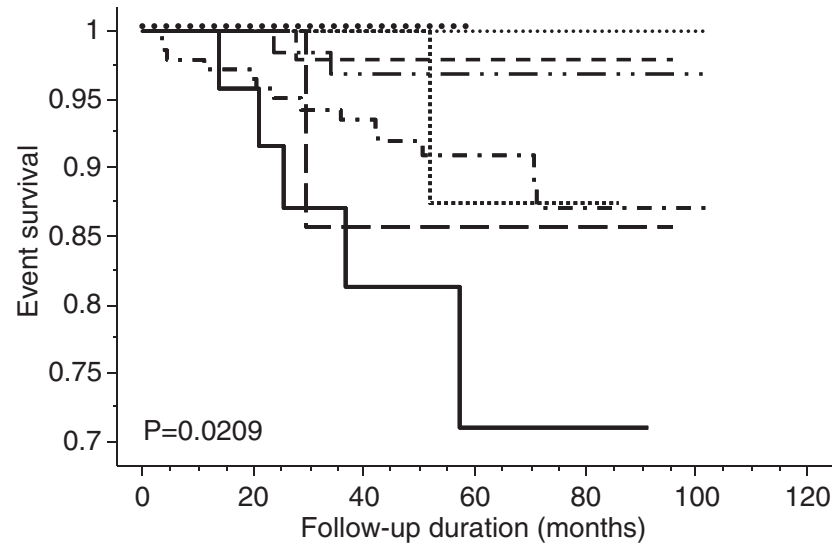

Figure 2 Kaplan-Meier analysis for stroke and CVD, combined analysis with $\beta_{1}$ Ser49Gly, $\beta_{2}$ Glu27GIn and $\beta_{3}$ Trp64Arg. ......... $\beta_{1}$ (SerGly+ GlyGly) $+\beta_{2} \quad$ GlnGIn $+\beta_{3} \quad$ (ArgArg+TrpArg); - - $\quad \beta_{1} \quad$ (SerGly+GlyGly) $+\beta_{2}$ GInGIn+ $\beta_{3} \operatorname{TrpTrp} ; \cdots \cdot \beta_{1}$ (SerGly+GlyGly) $+\beta_{2}$ GInGlu+ $\beta_{3}$ (ArgArg+TrpArg); ........ $\beta_{1}$ (SerGly+GlyGly) $+\beta_{2}$ GInGlu+ $\beta_{3}$ TrpTrp; $\quad---\beta_{1}$ SerSer $+\beta_{2}$ GInGIn $+\beta_{3}$ (ArgArg+TrpArg); - - $-\beta_{1}$ SerSer $+\beta_{2}$ GInGIn+ $\beta_{3} \operatorname{TrpTrp} ;--\beta_{1}$ SerSer $+\beta_{2}$ GInGlu $+\beta_{3}$ (ArgArg+TrpArg); $-\beta_{1}$ SerSer $+\beta_{2}$ GInGlu $+\beta_{3}$ TrpTrp.

reflected by high plasma norepinephrine levels. ${ }^{18}$ Dietary sodium restriction blunts the increase in nitric oxide-mediated $\beta_{2} A D R$ responsiveness of the forearm in Gly16 homozygotes following administration of normal dietary sodium, whereas baseline cardiac output decreases and peripheral resistance increases under sodium restriction. ${ }^{19}$ In male twins with highly similar genetic and environmental backgrounds, the Arg64 variant of the $\beta_{3} A D R$ polymorphism was found to be associated with insulin resistance and higher post-prandial hyperglycemia. $^{20}$

As we noted in the introduction, many cross-sectional studies have focused on cardiovascular-related disease and these SNPs of the $\beta A D R$ s. Moreover, previous reports have suggested that $\beta A D R s$ mediate smooth muscle relaxation in small resistance arteries and large conduit arteries. ${ }^{21}$ We previously found an association between a genetic polymorphism in Ser49Gly of the $\beta_{1} A D R$ and aortic stiffness as measured by pulse wave velocity, and the Gly49Gly genotype showed a genetic association with nitroglycerin-induced hyperemia. ${ }^{7}$ Although functional analysis is required, the Ser49Gly polymorphism of $\beta_{1} A D R$ might influence arterial functional changes. The Glu27Gln polymorphism of $\beta_{2} A D R$ has been identified by both electrocardiography and echocardiography to be significantly associated with left ventricular hypertrophy. Therefore, it is expected that these SNPs would affect prognostic outcomes of cardiovascular events in patients with essential hypertension.

There have been several reports about the prognostic impacts of Trp64Arg. In a multicenter study conducted in Spain, the Trp/Arg allele was associated with more severe insulin resistance, ${ }^{22}$ and the Arg allele was associated with a higher incidence of cardiac diseases in a Chinese population as determined by an 8 -year prospective cohort study of type 2 diabetes. ${ }^{23}$ The results from these studies are different from those of our present investigation; however, the baseline disease states and ethnicities were different. A large number of cohort studies of hypertensive patients are required to confirm our results.

We also performed a combined Kaplan-Meier analysis of the various polymorphisms, which showed an association for three SNPs with cardiovascular prognosis. Ser49Gly $\beta_{1} A D R$, Glu27Gln $\beta_{2} A D R$ and Trp64Arg $\beta_{3} A D R$ SNPs showed an additive effect on stroke and cardiovascular events in patients with essential hypertension. Although 
the present study had several limitations, described below, these results nonetheless underscore the possibility of the independent effects of these three $\beta A D R$ SNPs. These results do not directly impact common clinical practice; however, these findings might be important when considering the relationship between autonomic nervous system responses and hypertensive complications in patients with essential hypertension.

\section{Study limitations}

Our study was a hospital-based investigation that included patients with essential hypertension and had several limitations. First, a large number of cohort studies will be required to fully analyze the relationship between polymorphisms and cardiovascular events. Moreover, for combined analyses, the number of participants in this study was too small to yield definitive results. Furthermore, the participants were heterogeneous with respect to clinical background, which could influence cardiovascular events. For example, some patients were taking medications, and the patients had been sick for different periods of time. Finally, there may have been a selection bias because our study was a hospital-based study. In spite of these important limitations, the results showed a prognostic impact of $\beta A D R$ gene polymorphisms on cardiovascular events in hypertensive patients.

\section{ACKNOWLEDGEMENTS}

Grants: This study was funded by the Research Foundation for Community Medicine Research Meeting on Hypertension and Arteriosclerosis and by the Japan Heart Foundation for Research on Hypertension and the Autonomic Nervous System. We thank Kazuko Iwasa and Eriko Nagata for their technical and administrative assistance.

1 Walston J, Lowe A, Silver K, Yang Y, Bodkin NL, Hansen BC, Shuldiner AR. The beta3adrenergic receptor in the obesity and diabetes prone rhesus monkey is very similar to human and contains arginine at codon 64. Gene 1997; 188: 207-213.

2 Johnson M. The beta-adrenoceptor. Am J Respir Crit Care Med 1998; 158 (Part 3): S146-S153

3 Krum H. Sympathetic activation and the role of beta-blockers in chronic heart failure. Aust N Z J Med 1999; 29: 418-427.

4 Bohm M, Flesch M, Schnabel P. Beta-adrenergic signal transduction in the failing and hypertrophied myocardium. J Mol Med (Berlin, Germany) 1997; 75: 842-848.

5 Scarpace PJ, Tumer N, Mader SL. Beta-adrenergic function in aging. Basic mechanisms and clinical implications. Drugs Aging 1991; 1: 116-129.

6 Johnson JA, Terra SG. Beta-adrenergic receptor polymorphisms: cardiovascular disease associations and pharmacogenetics. Pharm Res 2002; 19: 1779-1787.

7 Yuan M, Ohishi M, Ito N, Sugimoto K, Takagi T, Terai M, Katsuya T, Rakugi H, Wu Z, Ogihara T. Genetic influences of beta-adrenoceptor polymorphisms on arterial functional changes and cardiac remodeling in hypertensive patients. Hypertens Res 2006; 29: 875-881.
8 Iwai C, Akita H, Kanazawa K, Shiga N, Terashima M, Matsuda Y, Takai E, Miyamoto Y, Shimizu M, Kajiya T, Hayashi T, Yokoyama M. Arg389Gly polymorphism of the human betal-adrenergic receptor in patients with nonfatal acute myocardial infarction. Am Heart J 2003; 146: 106-109.

9 Svetkey LP, Timmons PZ, Emovon O, Anderson NB, Preis L, Chen YT. Association of hypertension with beta2- and alpha2c10-adrenergic receptor genotype. Hypertension 1996; 27: 1210-1215.

10 Fujisawa T, Ikegami H, Yamato E, Takekawa K, Nakagawa Y, Hamada Y, Oga T, Ueda H, Shintani M, Fukuda M, Ogihara T. Association of Trp64Arg mutation of the beta3-adrenergic-receptor with NIDDM and body weight gain. Diabetologia 1996; 39: 349-352.

11 Wang Y, Luk AOY, Ma RCW, So WY, Tam CHT, Ng MCY, Yang X, Baum L, Lam V, Tong PCY, Chan JCN. Independent predictive roles of eotaxin Ala23Thr, paraoxonase 2 Ser311Cys and 33 -adrenergic receptor Trp64Arg polymorphisms on cardiac disease in Type 2 Diabetes - an 8-year prospective cohort analysis of 1297 patients. Diabet Med 2010; 27: 376-383.

12 Terai M, Ohishi M, Ito N, Takagi T, Tatara Y, Kaibe M, Komai N, Rakugi H, Ogihara T. Comparison of arterial functional evaluations as a predictor of cardiovascular events in hypertensive patients: the Non-Invasive Atherosclerotic Evaluation in Hypertension (NOAH) study. Hypertens Res 2008; 31: 1135-1145.

13 Bengtsson K, Melander O, Orho-Melander M, Lindblad U, Ranstam J, Rastam L, Groop L. Polymorphism in the beta(1)-adrenergic receptor gene and hypertension. Circulation 2001; 104: 187-190.

14 Okumura $\mathrm{K}$, Matsui $\mathrm{H}$, Ogawa $\mathrm{Y}$, Takahashi R, Matsubara $\mathrm{K}$, Imai H, Imamura A, Mizuno T, Tsuzuki M, Kitamura Y. The polymorphism of the beta3-adrenergic receptor gene is associated with reduced low-density lipoprotein particle size. Metabolism 2003; 52: 356-361.

15 Yoshida N, Nishimaki Y, Sugiyama M, Abe T, Tatsumi T, Tanoue A, Hirasawa A, Tsujimoto G. SNP genotyping in the beta(2)-adrenergic receptor by electronic microchip assay, DHPLC, and direct sequencing. J Hum Genet 2002; 47: 500-503.

16 Ranade K, Jorgenson E, Sheu WH, Pei D, Hsiung CA, Chiang FT, Chen YD, Pratt R, Olshen RA, Curb D, Cox DR, Botstein D, Risch N. A polymorphism in the beta1 adrenergic receptor is associated with resting heart rate. Am J Hum Genet 2002; 70: 935-942.

17 Mason DA, Moore JD, Green SA, Liggett SB. A gain-of-function polymorphism in a Gprotein coupling domain of the human betal-adrenergic receptor. J Biol Chem 1999; 274: 12670-12674.

18 Masuo K, Katsuya T, Kawaguchi H, Fu Y, Rakugi H, Ogihara T, Tuck ML. Rebound weight gain as associated with high plasma norepinephrine levels that are mediated through polymorphisms in the beta2-adrenoceptor. Am J Hypertens 2005; 18: 1508-1516.

19 Eisenach JH, Schroeder DR, Pike TL, Johnson CP, Schrage WG, Snyder EM, Johnson BD, Garovic VD, Turner ST, Joyner MJ. Dietary sodium restriction and beta2-adrenergic receptor polymorphism modulate cardiovascular function in humans. J Physiol 2006; 574 (Part 3): 955-965.

20 Hojlund K, Christiansen C, Bjornsbo KS, Poulsen P, Bathum L, Henriksen JE, Lammert $\mathrm{O}$, Beck-Nielsen $\mathrm{H}$. Energy expenditure, body composition and insulin response to glucose in male twins discordant for the Trp64Arg polymorphism of the beta3-adrenergic receptor gene. Diabetes Obes Metab 2006; 8: 322-330.

21 Kuusela TA, Jartti TT, Tahvanainen KU, Kaila TJ. Effects of terbutaline on peripheral vascular resistance and arterial compliance. J Cardiovasc Pharmacol 2004; 44: 74-81.

22 de Luis DA, Ballesters M, Riz E, Munoz C, Panacho A, Iglesias P, Lopez Guzman A, Maldonado A, Cordero M, San Martin L, Puigdevall V, Romero E, Gonzalez Sagrado M, Izaola O, Conde R. Polymorphism Trp64Arg of beta 3 adrenoreceptor gene: allelic frequencies and influence on insulin resistance in a multicenter study of Castilla-Leon. Nutr Hosp 2010; 25: 299-303.

23 Wang Y, Luk AO, Ma RC, So WY, Tam CH, Ng MC, Yang X, Baum L, Lam V, Tong PC, Chan JC. Independent predictive roles of eotaxin Ala23Thr, paraoxonase 2 Ser311Cys and beta-adrenergic receptor Trp64Arg polymorphisms on cardiac disease in Type 2 Diabetes-an 8-year prospective cohort analysis of 1297 patients. Diabet Med 2010; 27: $376-383$. 